\title{
A cost-effective ROF system employing FBG and DML to generate an optical mm-wave
}

\author{
Viet Hoang Nguyen, ${ }^{*}{ }^{1,2}$ Cheng Huang ${ }^{1}$ \\ ${ }^{1}$ School of Computer and Communication, Hunan University, Changsha 410082, China, \\ ${ }^{2}$ Faculty of Electronics, Ho Chi Minh City University of Industry, Ho Chi Minh City, Vietnam
}

Received June 05, 2013; accepted September 25, 2013; published September 30, 2013

\begin{abstract}
We have investigated and experimentally demonstrated a ROF system employing FBG and low-cost DML to generate an optical mm-wave. We employ FBG because the bandwidth of the optical modulator is largely reduced and the architecture of the ROF system is simpler. Because no expensive broadband external modulator is used, the overall system is considered as a cost-effective solution. This novel scheme employs low-cost DML to generate an mm-wave signal which is a practical scheme to be applied for future broadband access networks.
\end{abstract}

Recently ROF techniques have become attractive solutions in realizing future broadband wireless networks to provide large capacity and high flexibility [1-5] because they can be used for the distribution of wireless signals which grow very fast. To reduce the total cost and make simpler configuration of the ROF system sand connect as many users as possible, novel schemes of wavelength reuse or centralized light-waves in the $\mathrm{CO}$ have been proposed and experimentally demonstrated in [6-9]. To realize optical millimeter-wave generation, many techniques have been reported, such as the frequency up-conversions using four-wave mixing [10], cross-gain modulation in a semiconductor amplifier [1113], and frequency doubling using optical carrier suppression modulation [14-15]. When compared with an optical external modulator, the DML is cost- efficient and has a higher output power. For $40 \mathrm{GHz}$ optical mm-wave signal generation, only $20 \mathrm{GHz}$ optical and electrical components are required, because the generated optical mm-wave signal will be up to $40 \mathrm{GHz}$ after it is separated from the optical carrier by using FBG [16]. We employ FBG because the bandwidth of the optical modulator is largely reduced and the architecture of the ROF system is simpler. The ROF system which based on DML to generate an optical mm-wave carrying OOK signal has been introduced [17]. In this letter, we have investigated and experimentally demonstrated an ROF system employing FBG and low-cost DML to generate an optical mm-wave. A theoretical demonstration analysis of the proposed system is also presented. It can be considered as a very simple and cost-effective solution because a low $\mathrm{RF}$ oscillator is employed to generate an optical mmwave signal with frequency duplication.

*E-mail: viet02342000@yahoo.com
Table 1

ROF system parameters

\begin{tabular}{lc}
\hline \hline \multicolumn{1}{c}{ Parameters } & Requirement/Result \\
\hline mm-wave generation & DSB modulation \\
Optical filter & FBG \\
External modulator & Don't need \\
Transmission length & $40 \mathrm{~km}$ \\
Power penalty & $2.1 \mathrm{~dB}$ \\
\hline \hline
\end{tabular}

Table 1 shows the ROF system parameters. In this system, the expensive broadband external modulator is removed. We employ a high-frequency electrical mixer to generate RF signals which are employed to drive the DML to generate a DSB optical mm-wave.

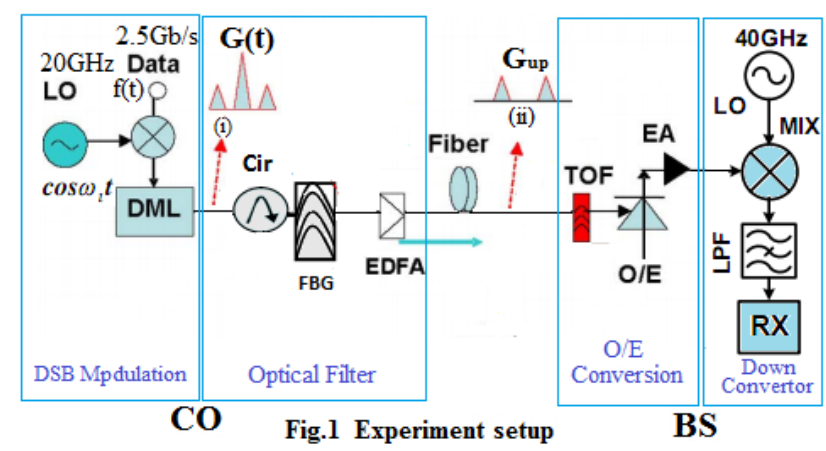

In the real system, there are some higher harmonics because of the nonlinearity of the DML, but they are too small to be considered. The $2.5 \mathrm{~Gb} / \mathrm{s}$ baseband signal is mixed with a $20 \mathrm{GHz}$ sinusoidal wave to realize sub-carrier modulation (SCM) and then the mixed signals are employed to drive DML to generate DSB optical signals. The waveform output of the DML which is driven by a mixing RF signal $f(t) \cos \omega_{1} t$ can be approximately represented by $\mathrm{G}(\mathrm{t})$ which has an optical carrier and the two first-order sidebands as follows:

$$
\begin{aligned}
\mathrm{G}(\mathrm{t}) \cong & \mathrm{G}_{0} \mathrm{~J}_{1}[\xi \mathrm{f}(\mathrm{t})] \cos \left(\omega_{\mathrm{c}}+\omega_{1}\right) \mathrm{t}+\mathrm{G}_{0} \mathrm{~J}_{0}[\xi \mathrm{f}(\mathrm{t})] \cos \omega_{\mathrm{c}} \mathrm{t}+ \\
& +\mathrm{G}_{0} \mathrm{~J}_{1}[\xi \mathrm{f}(\mathrm{t})] \cos \left(\omega_{\mathrm{c}}-\omega_{1}\right) \mathrm{t}
\end{aligned}
$$

where $\mathrm{f}(\mathrm{t})[=0$, or 1$]$ is the digital downlink data, $\omega_{1}$ is the angular frequency of LO signal. $G_{0}$ and $\omega_{c}$ are the electrical amplitude and angular frequency of the lightwave output of DML, respectively. $\mathrm{J}_{0}($.$) and \mathrm{J}_{1}($.$) are$ 
the zero and the first-orders of the Bessel function of the first kind, respectively, and $\xi$ is the intensity modulation index.

As shown in Fig. 1, an optical circulator (Cir) and an FBG are employed to suppress the optical carrier from the first-order sidebands. To simplify the analysis, we assumed that the optical carrier was completely separated from the first-order optical sidebands.

For transmission over the L-length downlink fiber, $\beta(\omega)$ represents the propagation constant of the fiber. $\tau_{+}$and $\tau_{-}$ are the delay deviations caused by the fiber chromatic dispersion at the optical sidebands $\left(\omega_{\mathrm{c}}+\omega_{1}\right.$ and $\left.\omega_{\mathrm{c}}-\omega_{1}\right)$ from the original optical carrier at $\omega_{c}$, respectively They can be expressed as $\tau_{+}=-2 \pi \mathrm{LcD} \omega_{1} / \omega_{\mathrm{c}}{ }^{2}$ and $\tau_{-}=2 \pi \mathrm{LcD}$ $\omega_{1} / \omega_{\mathrm{c}}{ }^{2}$, where $\mathrm{D}$ is the chromatic dispersion parameter. The generated optical DSB signal transmitted over the L: length downlink fiber, due to the fiber chromatic dispersion, becomes:

$$
\begin{aligned}
& \mathrm{G}(\mathrm{t})=\mathrm{G}_{0} \mathrm{~J}_{1}\left[\xi \mathrm{f}\left(\mathrm{t}-\tau_{+}\right)\right] \cos \left[\left(\omega_{\mathrm{c}}+\omega_{1}\right) \mathrm{t}-\beta\left(\omega_{\mathrm{c}}+\omega_{1}\right) \mathrm{L}\right]+ \\
& +\mathrm{G}_{0} \mathrm{~J}_{1}\left[\xi \mathrm{f}\left(\mathrm{t}-\tau_{-}\right)\right] \cos \left[\left(\omega_{\mathrm{c}}-\omega_{1}\right) \mathrm{t}-\beta\left(\omega_{\mathrm{c}}-\omega_{1}\right) \mathrm{L}\right] \\
& +\mathrm{G}_{0} \mathrm{~J}_{0}[\xi \mathrm{f}(\mathrm{t})] \cos \left[\omega_{\mathrm{c}} \mathrm{t}-\beta\left(\omega_{\mathrm{c}}\right) \mathrm{L}\right] \\
& \quad \mathrm{G}_{\mathrm{up}}=\mathrm{G}_{0} \mathrm{~J}_{1}\left[\xi \mathrm{f}\left(\mathrm{t}-\tau_{+}\right)\right] \cos \left[\left(\omega_{\mathrm{c}}+\omega_{1}\right) \mathrm{t}-\beta\left(\omega_{\mathrm{c}}+\omega_{1}\right) \mathrm{L}\right]+ \\
& +\mathrm{G}_{0} \mathrm{~J}_{1}\left[\xi \mathrm{f}\left(\mathrm{t}-\tau_{-}\right)\right] \cos \left[\left(\omega_{\mathrm{c}}-\omega_{1}\right) \mathrm{t}-\beta\left(\omega_{\mathrm{c}}-\omega_{1}\right) \mathrm{L}\right]
\end{aligned}
$$

Applying this upper branch optical signal to a broadband photo-detector (PD), due to square-law detection by the PD, the two terms in Eqs. (2) beat with each other and generate an electrical signal as follows:

$$
\begin{gathered}
\mathrm{i}_{\text {out }}=\frac{1}{2} G_{0}^{2} J_{1}^{2}(\xi)\left[\mathrm{f}\left(\mathrm{t}-\tau_{+}\right)+\mathrm{f}\left(\mathrm{t}-\tau_{-}\right)\right]+G_{0}^{2} J_{1}^{2}(\xi) \quad \mathrm{f}\left(\mathrm{t}-\tau_{+}\right) \quad \mathrm{f}\left(\mathrm{t}-\tau_{-}\right) \\
\cdot \cos \left[2 \omega_{1} \mathrm{t}-\beta\left(\omega_{\mathrm{c}}+\omega_{1}\right) \mathrm{L}+\beta\left(\omega_{\mathrm{c}}+\omega_{1}\right) \mathrm{L}\right] .
\end{gathered}
$$

We have: $\beta\left(\omega_{\mathrm{c}} \pm \omega_{1}\right)=\beta\left(\omega_{\mathrm{c}}\right) \pm \omega_{1} \beta^{\prime}\left(\omega_{\mathrm{c}}\right)+\frac{1}{2} \omega_{1}^{2} \beta^{\prime \prime}\left(\omega_{\mathrm{c}}\right)$ $+\ldots$

and the property of $\mathrm{J}_{1}(\xi \chi)=\chi \mathrm{J}_{1}(\xi)$ (for $\chi=0$, or 1 ) is used. By expanding the propagation constant of the fiber for each optical sideband to a Taylor series around the angular frequency of the optical carrier, the generated electrical signal becomes:

$$
\begin{gathered}
\mathrm{i}_{\text {out }}=\frac{1}{2} G_{0}^{2} J_{1}^{2}(\xi)\left[\mathrm{f}\left(\mathrm{t}-\tau_{+}\right)+\mathrm{f}\left(\mathrm{t}-\tau_{-}\right)\right]+G_{0}^{2} J_{1}^{2}(\xi) \mathrm{f}\left(\mathrm{t}-\tau_{+}\right) \mathrm{f}\left(\mathrm{t}-\tau_{-}\right) \\
\cdot \cos 2 \omega_{1}\left[\mathrm{t}-\beta^{\prime}\left(\omega_{\mathrm{c}}\right) \mathrm{L}\right]
\end{gathered}
$$

It can be seen from Eq. (3) that the generated electrical signal mainly consists of a DC component $\frac{1}{2} G_{0}^{2} J_{1}^{2}(\xi)[\mathrm{f}(\mathrm{t}-$ $\left.\left.\tau_{+}\right)+\mathrm{f}\left(\mathrm{t}-\tau_{-}\right)\right]$and a harmonic component at $2 \omega_{1}$. Downconverted from the mm-wave, the duty cycle of the data code will be reduced. When $\tau=\tau_{-}-\tau_{+}$is equal to the duty cycle of the baseband data, there will be no mm-wave signal at $2 \omega_{1}$, where $\tau$ is the delay time difference between the first-order upper and lower optical sidebands caused by chromatic dispersion. The transmission distance of the optical mm-wave generated by this scheme is also limited because of the interference to the baseband data.
The experiment set up is shown in Fig.1. In the CO, we employ a commercial DML with a central wavelength of $1550 \mathrm{~nm}$ biased at $57 \mathrm{~mA}$ to generate a continuous lightwave $(\mathrm{CW})$. The output power of the DML biased at $57 \mathrm{~mA}$ was $4 \mathrm{dBm}$, and the $3-\mathrm{dB}$ bandwidth of the DML was larger than $25 \mathrm{GHz}$. The RF signals were generated by using an electrical mixer to combine the $20-\mathrm{GHz} \mathrm{RF}$ clock (sinusoidal wave) and $2.5-\mathrm{Gb} / \mathrm{s}$ electrical signal (downstream data) with a PRBS length of $2^{31}-1$. Then, the mixed RF signals were boosted to $2.7 \mathrm{Vp}$-p to drive the DML. The optical spectrum after modulation is shown in Fig. 2 as inset (i). We can see that the second-order sideband of each channel is around $20 \mathrm{~dB}$ lower than the first-order sideband. Thus, the second-order sidebands have little effect on the transmission of the optical mmwave signals in the SMF fiber. A Cir and a FBG are used to suppress the optical carrier from the first-order sidebands of the generated optical DSB signal. The FBG has a $3 \mathrm{~dB}$ reflection bandwidth of $0.2 \mathrm{~nm}$. Then the optical signals will be transmitted over the $40-\mathrm{km}$ SSMF to the BS after boosting the power by an erbium-doped fiber amplifier (EDFA). In the BS, optical signals are detected by an optical receiver which is a high-speed photodetector with a $3 \mathrm{~dB}$ bandwidth of $50 \mathrm{GHz}$. The optical spectrum of the first-order sidebands is shown in Fig. 2 as inset (ii). We can see that the first-order optical side-band is around $15 \mathrm{~dB}$ larger than the optical carrier. Thus, the residual optical carrier has a negligible effect on the performance of the optical mm-wave signals. The repetitive frequency of the first-order signal is $40 \mathrm{GHz}$.
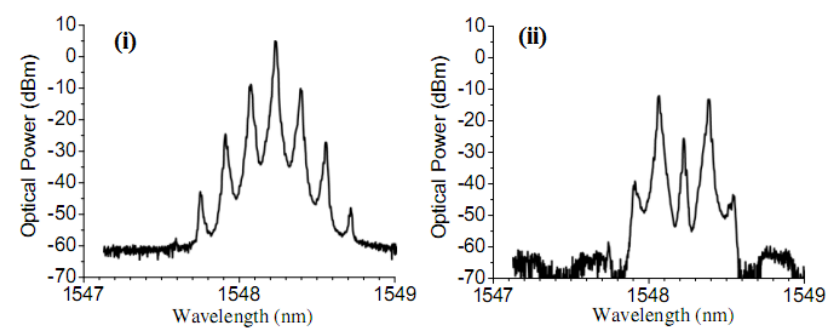

Fig.2 Optical spectra at different location in Fig.1

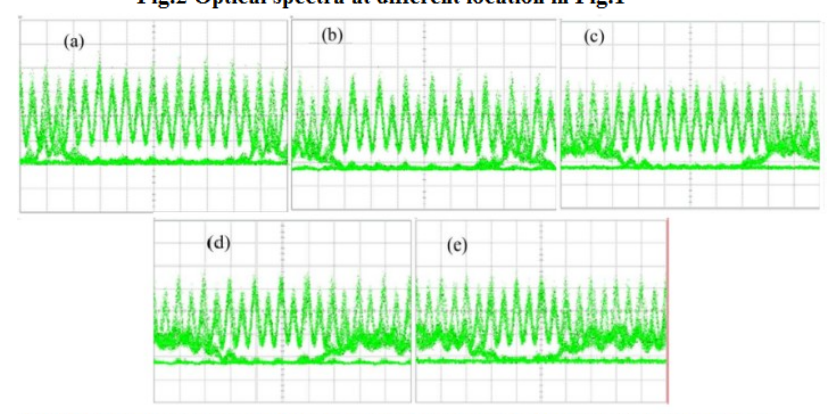

Fig.3 Eye diagrams (100ps/div) of signals with different transmission distances: (a) $0 \mathrm{~km}$, (b) $10 \mathrm{~km}$, (c) $20 \mathrm{~km}$, (d) $30 \mathrm{~km}$ and (e) $40 \mathrm{~km}$

Figures 3(a)-3(e) show the eye diagrams of signals after transmission over different distances. The slope of the signal code edge becomes flat as the distance is increased 
due to fiber dispersion. Figure 3(e) shows that the signals performance is still clear after optical mm-wave signals were transmitted over $40 \mathrm{~km}$. The optical signals were filtered by a TOF with a bandwidth of $0.5 \mathrm{~nm}$ before $\mathrm{O} / \mathrm{E}$ conversion via a PIN PD with a $3-\mathrm{dB}$ bandwidth of 60 $\mathrm{GHz}$. Then, the converted signals were boosted by an electrical amplifier (EA) with a bandwidth of $10 \mathrm{GHz}$ centered at $40 \mathrm{GHz}$. An electrical LO signal at $40 \mathrm{GHz}$ was generated by using a frequency multiplier from 10 to $40 \mathrm{GHz}$. We used the electrical LO signal and a mixer to down-convert the mm-wave signal. The down-converted $2.5 \mathrm{Gbit} / \mathrm{s}$ signal was detected by a BER tester before it was filtered by a low pass electrical filter with a bandwidth of $2.8 \mathrm{GHz}$.

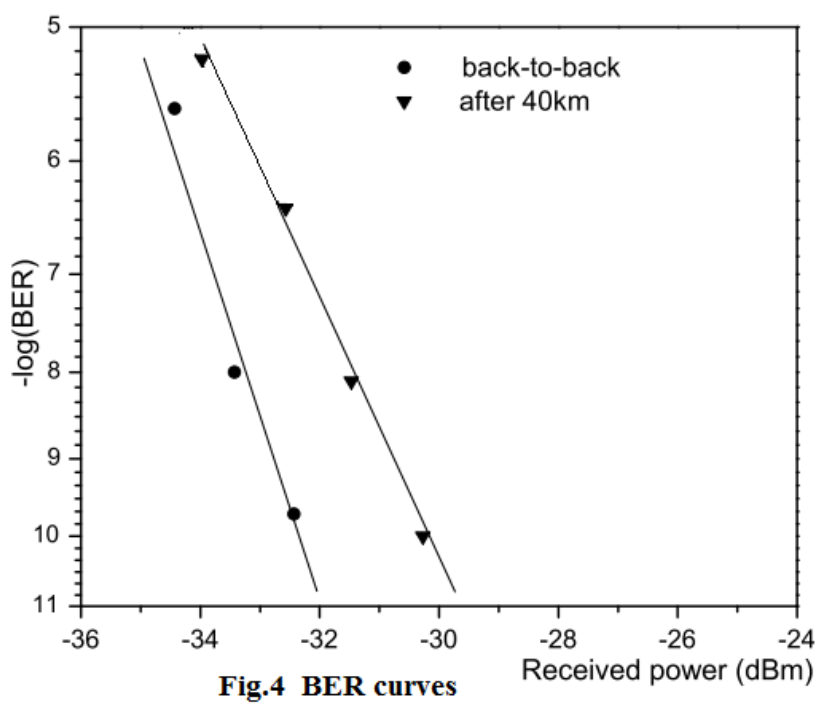

The measured BER performance is shown in Fig. 4. The power penalty is $2.1 \mathrm{~dB}$, mainly caused by the $40-\mathrm{km}$ SMF-28 dispersion. The data were carried by two firstorder sidebands with a frequency spacing of $40 \mathrm{GHz}$. Because of the chromatic dispersion of SMF, the firstorder upper- and lower sidebands will have different group velocity dispersion and result in a delay time difference between two sidebands at the receivers. The dispersion leads to the reduction of optical eye-opening and causes a power penalty. Thus, data signals will be degraded after increasing the transmission distance, showing the same results as in theoretical analysis.

In conclusion, we have investigated and experimentally demonstrated a ROF system employing FBG and lowcost DML to generate an optical mm-wave. The doublefrequency mm-wave is generated by beating the two firstorder sidebands of the optical DSB signal, which is generated via a broadband DML driven by mixing RF signals. We employ FBG because the repetitive frequency of the RF source and the bandwidth of the optical modulator are largely reduced and the architecture of the ROF system is simpler. The theoretical analysis clarifies how the transmission distance of the optical mm-wave generated by this scheme is also limited because of the interference to the baseband data. The measured BER performance results in the power penalty of $2.1 \mathrm{~dB}$, which is mainly caused by the $40-\mathrm{km}$ SMF-28 dispersion. The data signals will be degraded after increasing the transmission distance, with the same results as in the theoretical analysis. Because no expensive broadband external modulator is used, the overall system is very simple, being considered a cost-effective solution. Thus, we believe this novel scheme is practical and suitable for future application in broadband access networks. One of the main applications is the distributed antenna system (DAS). In a DAS, RoF transmission links are employed to connect a CU to many remote antenna units, in order to provide high capacity for short range communications in areas such as city centers, office blocks, shopping malls and transport hubs.

\section{References}

[1] L. Xu, T. Wang, in Proc. ICO, 1 (2011)

[2] S. Mikel, L. Alayn, Opt. Expr. 18(22), 22906 (2010).

[3] Z. Cao, J. Yu, M. Xia, Q. Tang, Y. Gao, W. Wang, L. Chen, J. Lightw. Technol. 28(16), 2423 (2010).

[4] J. Yu et al., J. Lightw. Technol. 28(16), 2376 (2010)

[5] C. Tsekrekos, T. Kuri, K. Kitayama, J. Lightw. Technol. 28(19), 2783 (2010)

[6] Z. Dong, J. Lu, Y. Pi, X. Lei, L. Chen, J. Yu, J. Opt. Network. 7, $736(2008)$

[7] W. Jiang, C.T. Lin, A. Ng'oma, P. T. Shih, J. Chen, M. Sauer, F. Annunziata, S. Chi, J Lightw. Technol. 28(16), 2238 (2010).

[8] J. Ma, J. Opt. Commun. Netw. 3(2), 127 (2011).

[9] Z. Cao, J. Yu, H. Zhou, W. Wang, M. Xia, J. Wang, Q. Tang, L. Chen, J. Opt. Commun. Netw. 2(2), 117 (2010).

[10] J. Yu, J. Gu, X. Liu, Z. Jia, G. Chang, IEEE Photon. Technol. Lett. 17, 1986 (2005)

[11] J. Seo, Y. Seo, W. Choi, IEEE Photon. Technol. Lett. 18, 1389 (2005).

[12] J. Yu, Z. Jia, G. Chang, IEEE Photon. Technol. Lett. 18, 2421 (2005).

[13] H. Song, J. Lee, IEEE Photon. Technol. Lett. 17, 1731 (2005).

[14] J. Yu, Z. Jia, L. Yi, Y. Su, G. Chang, IEEE Photon. Technol. Lett. 18, 265 (2006).

[15] J. Yu, Z. Jia, L. Xu, L.Chen, T. Wang, G.K, Chang, IEEE Photon. Technol. Lett. 18, 1418 (2006).

[16] M. Attygalle, C. Lim, G. Pendock, A. Nirmalathas, G. Edvell, IEEE Photon. Technol. Lett. 17, 190 (2005).

[17] L. Chen, J. He, Y. Li, H. Wen, Y. Shao, C. Huang, L. Hu, Y. Pi, Z. Dong, Y. Li, X. Lei, S. Wen, ECOC 2, 45 (2007). 